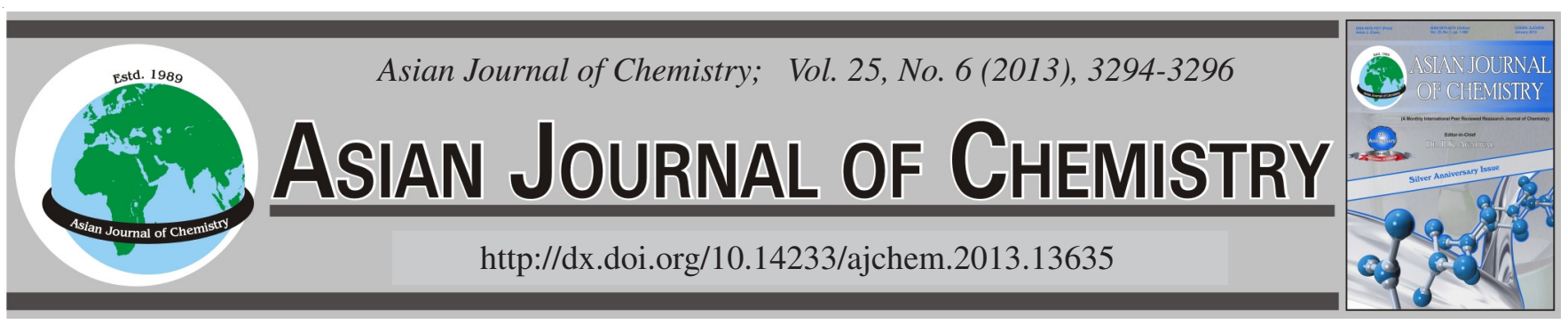

\title{
Synthesis and Bioactivity of Furoxan-Based Nitric Oxide- Releasing Colchicine Derivatives as Anticancer Agents
}

\author{
Li Hong Shen ${ }^{1, *}$, Sheng Li Wang ${ }^{1}$, Hong Yu Li ${ }^{1}$, Yi Sheng Lai ${ }^{2}$ and Li Jie Liu ${ }^{3}$
}

${ }^{1}$ Handan Key Laboratory of Organic Small Molecule Materials, Handan College, Handan 056005, P.R. China

${ }^{2}$ Center of Drug Discovery, China Pharmaceutical University, Nanjing 210009, P.R. China

${ }^{3}$ Hebei Yiling Medicine Corporation Ltd, Shijiazhuang 050035, P.R. China

*Corresponding author: E-mail: latishen@sohu.com

(Received: 21 February 2012;

Accepted: 17 December 2012)

AJC-12570

A series of novel nitric oxide-donating colchicine derivatives $(9 \mathrm{9a-j})$ were synthesized by coupling furoxan with N-methyl colchiceinamide
through an appropriate spacer arm and their cytotoxicity against four human cancer cell lines in vitro were evaluated by MTT method. It
was found that many of the derivatives displayed significant activity, particularly, compound 9f showed more potent cytotoxic activities
than colchicine.

\section{INTRODUCTION}

Colchicine (Col, 1), a major alkaloid extracted from the seed of Colchicum autumnale and Gloriosa superba, is a drug interfering with microtubule assembly both in vivo and in vitro, thereby causing cells to accumulate in mitotic arrest during the cell cycle ${ }^{1,2}$. Colchicine has antiinflammatory, antimitotic and antifibrotic activity ${ }^{3}$. Colchicine also finds applications in various other diseases like pseudogout, familial Mediterrenian fever, tumor growth, cirrhosis of the liver and bile and amyloidosis ${ }^{4,5}$. Although colchicine is a potent antimitotic agent, its medicinal uses are limited due to its high toxicity. Therefore, many attempts have been made to discover more effective and less toxic analogues of colchicine by modifying the substituents of its basic structure ${ }^{6}$. N-Methyl colchiceinamide (2, SchemeI), a synthetic derivative of colchicine, wherein the 10-methoxy group is replaced by a 10-methylimino group, showed considerably higher stability toward acid hydrolysis and is a slightly less active antitumor and toxicity agent than colchicine ${ }^{4}$.

Nitric oxide (NO), naturally synthesized from L-arginine by the action of NO synthase (NOS), is a small, diffusible, highly reactive molecule involved in the regulation of many physiological processes including blood vessel dilatation, neurotransmission and events of the immune system. Nitric oxide can also be generated from synthetic NO-releasing compounds, such as nitrate, furoxan, hydroxyguanidine, Snitrosothiol, diazeniumdiolate and others ${ }^{7,8}$. Studies showed that high concentration of NO was cytotoxic and could induce the apoptosis of tumor cells, prevent tumors from metastatizing and assist macrophage to kill tumor cells ${ }^{9}$. During recent years, NO-releasing derivatives have currently come into focus on the treatment of cancer, inflammation and vascular diseases ${ }^{10,11}$.

Chang and his co-workers reported a group of nitrate derivatives of colchicine, which have proved that the structure modification of the 10 position does not interfere with the molecular recognition of colchicine ${ }^{12}$. In our design, furoxans were substituted for organic nitrates acting as NO donors, because furoxans are supposed to be able to release higher concentrations of $\mathrm{NO}$ in vivo and can also effectively avoid "the nitrate tolerance"13. By releasing NO in vivo, we hope to enhance the antitumor activity of these derivatives and particularly to minimize the side effects.

The synthetic route of these target compounds is outlined in Scheme-I. Colchicine (1) was purchased from Nanjing Tianzun Chemicals Co. Ltd. China, with an over $98 \%$ purity. The lead compound $\mathbf{3}$ was prepared from $\mathbf{1}$, according to the literature $^{14}$ in $85 \%$ yield.

The synthesis of furoxans $\mathbf{7 a - 7} \mathbf{j}$ utilized thiophenol $\mathbf{4}$ as the starting material, which was converted to 2-(phenylthio)acetic acid 5 by treatment with chloroacetic acid in $97 \%$ yield. Compound $\mathbf{5}$ was oxidized by $30 \% \mathrm{H}_{2} \mathrm{O}_{2}$ solution, followed by treatment with fuming $\mathrm{HNO}_{3}$ to offer diphenylsulfonylfuroxan 6. Reaction of $\mathbf{6}$ with corresponding diols gave $7 \mathbf{a}-7 \mathbf{j}$ with the yields of $65-85 \%$. 
<smiles>COc1cc2c(c(OC)c1OC)-c1ccc(OC)c(=O)cc1CCC2NC(C)=O</smiles><smiles>CNc1ccc2c(cc1=O)CCCc1cc(OC)c(OC)c(OC)c1-2</smiles><smiles>CNc1ccc2c(cc1=O)[C@H](N)CCc1cc(OC)c(OC)c(OC)c1-2</smiles><smiles></smiles>

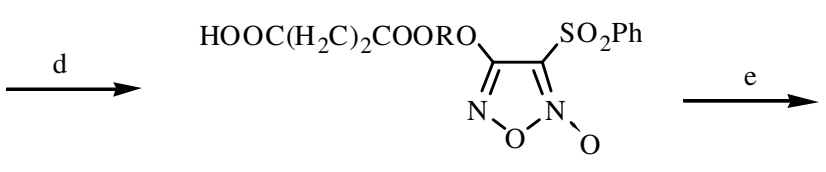<smiles>CNc1ccc2c(cc1=O)[C@@H](NC(=O)CC(=O)Oc1non1[O-])CCc1cc(OC)c(OC)c(OC)c1-2</smiles>

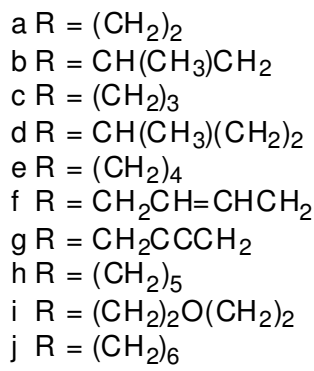

Scheme-I: Reagents and conditions: (a) $1 . \mathrm{NaOH}$ (aq.), $\mathrm{ClCH}_{2} \mathrm{COOH}, 140{ }^{\circ} \mathrm{C}, 2 \mathrm{~h} ; 2.6 \mathrm{~N} \mathrm{HCl}, 97 \%$; (b) $1.30 \% \mathrm{H}_{2} \mathrm{O}_{2}, \mathrm{AcOH}$, rt, $3 \mathrm{~h} ; 2$. fuming $\mathrm{HNO}_{3}, 90$ ${ }^{\circ} \mathrm{C}, 4$ h; (c) HOROH, THF, $25 \% \mathrm{NaOH}, \mathrm{rt}, 2 \mathrm{~h}, 65-85 \%$; (d) succinic anhydride, pyridine, $60{ }^{\circ} \mathrm{C}, 5 \mathrm{~h}, 90-95 \%$; (e) 3, EDC, $\mathrm{DMAP}, \mathrm{CH}_{2} \mathrm{Cl}_{2}, \mathrm{rt}^{\mathrm{r}}$, $24 \mathrm{~h}, 60-85 \%$

Compounds $7 \mathbf{a}-7 \mathbf{j}$ were acylated by succinic anhydride in dry pyridine at $60{ }^{\circ} \mathrm{C}$ to give succinates $\mathbf{8 a - 8 j}$ in $90-95 \%$ yield. Finally, 8a-8j were treated with 3 in $\mathrm{CH}_{2} \mathrm{Cl}_{2}$ in the presence of EDC and DMAP to give target compounds $\mathbf{9 a - 9} \mathbf{j}$ in good yields (60-85\%).

The resulting products were purified by column chromatography and their structures were shown in Scheme-I and the data of yield, MS, IR and ${ }^{1} \mathrm{H}$ NMR spectra and elemental analysis of selected compounds were shown in reference ${ }^{15}$.

The cytotoxic activity of all target compounds in vitro was determined by MTT assay ${ }^{16}$, using colchicine as a positive control and the result is summarized in Table-1. Four different cell lines were used: A2780 (human ovary cancer), A549

TABLE-1

CYTOTOXICITY DATA OF THE TARGET COMPOUNDS

\begin{tabular}{ccccc}
\hline \multirow{2}{*}{ Compound } & \multicolumn{4}{c}{$\mathrm{IC}_{50}(\mu \mathrm{M}) /$ cell line } \\
\cline { 2 - 5 } & $\mathrm{A} 2780$ & $\mathrm{~A} 549$ & $\mathrm{BEL} 7402$ & $\mathrm{MCF7}$ \\
\hline $\mathbf{9 a}$ & 0.104 & 0.106 & 0.102 & 0.103 \\
$\mathbf{9 b}$ & 0.098 & 0.087 & 0.080 & 0.135 \\
$\mathbf{9 c}$ & 0.110 & 0.082 & 0.093 & 0.078 \\
$\mathbf{9 d}$ & 0.096 & 0.086 & 0.079 & 0.100 \\
$\mathbf{9 e}$ & 0.109 & 0.105 & 0.081 & 0.095 \\
$\mathbf{9 f}$ & 0.045 & 0.035 & 0.012 & 0.008 \\
$\mathbf{9 g}$ & 0.097 & 0.088 & 0.099 & 0.076 \\
$\mathbf{9 h}$ & 0.096 & 0.079 & 0.083 & 0.088 \\
$\mathbf{9 i}$ & 0.106 & 0.115 & 0.180 & 0.159 \\
$\mathbf{9 j}$ & 0.100 & 0.106 & 0.130 & 0.104 \\
Colchicine & 0.094 & 0.078 & 0.080 & 0.084 \\
\hline
\end{tabular}

(human lung cancer), BEL7402 (human hepatoma), MCF7 (Human breast carcinoma).

The study results indicate that these novel nitric oxidereleasing derivatives showed superior or comparable cytotoxic activity to colchicine in vitro. For human ovary cancer cell line (A2780) and human lung cancer cell line (A549), all compounds exhibited weak inhibitory activity, except compound 9f. In human hepatoma cell line (BEL7402), compounds 9b, 9d, $9 \mathbf{e}$ and $9 \mathrm{~h}$ have similar cytotoxicity as colchicine, whereas compound 9f has more potent cytotoxicity than colchicine. As to human breast carcinoma cell line (MCF7), compound 9f exhibited almost tenfold potent activities than colchicine. The enhanced bioactivity suggested that the introduction of the furoxan group, which releases $\mathrm{NO}$, did play a cooperative role in the exertion of antitumor activity, leading to a better balance between NO- and colchicine-dependent activity in the derivatives.

According to these cytotoxicity studies, it is suggested that different length of spacer arm in the hybrid compounds did have an impact on the molecules' capability to inhibit cancer cell growth to various degrees, but without showing significant difference on regular pharmacological behaviours.

In summary, a series of furoxan-based NO-donating colchicine derivatives were synthesized and evaluated for their in vitro cytotoxicity against four human tumor cell lines. Among all the derivatives, compound 9 f showed the strongest inhibitory activity against all the tested cell lines and it is currently under our further investigation. 


\section{REFERENCES}

1. E. Niel, Joint Bone Spine, 73, 672 (2006).

2. B. Bhattacharyya, D. Panda, S. Gupta and M. Banerjee, Med. Res. Rev., 28, 155 (2008)

3. A.D. Kinghorn, B.N. Su, D.S. Jang, L.C. Chang, D. Lee, J.Q. Gu, E.J. Carcache-Blanco, A.D. Pawlus, S.K. Lee, E.J. Park, M. Cuendet, J.J. Gills, K. Bhat, H.S. Park, E. Mata-Greenwood, L.L. Song, M. Jang and J.M. Pezzuto, Planta Med., 70, 691 (2004)

4. M. Cifuentes, B. Schilling, R. Ravindra, J. Winter and M.E. Janik, Bioorg. Med. Chem. Lett., 16, 2761 (2006).

5. C. Cerquaglia, M. Diaco, G. Nucera, M. La Regina, M. Montalto and R. Manna, Curr. Drug Targets Inflamm. Allergy, 4, 117 (2005).

6. K.H. Lee, J. Nat. Prod., 67, 273 (2004)

7. P.G. Wang, M. Xian, X. Tang, X. Wu, Z. Wen, T. Cai and A.J. Janczuk, Chem. Rev., 102, 1091 (2002).

8. S. Pervin, G. Chaudhuri and R. Singh, Curr. Pharm. Des., 16, 451 (2010).

9. S. Mocellin, Curr. Cancer Drug Targets, 9, 214 (2009).

10. T. Min, B. Yi, P. Zhang et al., Med. Chem. Res., 18, 495 (2009).

11. T. Wang, Y.H. Zhang, X.W. Kong, Y.S. Lai, H. Ji, Y.P. Chen and S.X. Peng, Chem. Biodivers, 6, 466 (2009).

12. D.J. Chang, E.Y. Yoon, G.B. Lee, S.O. Kim, W.J. Kim, Y.M. Kim, J.W Jung, H. An and Y.G. Suh, Bioorg. Med. Chem. Lett., 19, 4416 (2009).

13. L. Fang, Y.H. Zhang, J. Lehmann, Y. Wang, H. Ji and D. Ding, Bioorg. Med. Chem. Lett., 17, 1062 (2007).

14. L.H. Shen, Y. Li, D.H. Zhang, Y.S. Lai and L.J. Liu, Chin. Chem. Lett., 22, 768 (2011)

15. The data of selected compounds: 9a: yield $60.0 \%, \mathrm{IR}\left(\mathrm{KBr}, \mathrm{v}_{\max }, \mathrm{cm}^{-1}\right)$ : 3436, 2929, 1736, 1623, 1553, 1450, 1387, 1170; ${ }^{1} \mathrm{H} \mathrm{NMR}\left(\mathrm{CDCl}_{3}\right.$, $300 \mathrm{MHz}, \delta \mathrm{ppm}): 1.50-3.38(\mathrm{~m}, 9 \mathrm{H}), 3.07\left(\mathrm{~d}, 3 \mathrm{H}, \mathrm{N}-\mathrm{CH}_{3}\right), 3.66(\mathrm{~s}, 3 \mathrm{H}$, MeO-1 ), 3.90 (s, 3H, MeO-2 ), 3.93 (s, 3H, MeO-3), 4.24-4.28 (t, $2 \mathrm{H}$ $\left.J=6.0 \mathrm{~Hz}, \mathrm{OCH}_{2}\right), 4.44-4.48\left(\mathrm{t}, 2 \mathrm{H}, J=6.0 \mathrm{~Hz}, \mathrm{OCH}_{2}\right), 4.62-4.72(\mathrm{~m}, 1 \mathrm{H}$, H-7), 6.52 (s, 1H, H-4), 7.30 (m, 2H, Ar-H), 7.49 (s, 1H), 7.60-7.66 (m, 2H, ArH), 7.74-7.77 (m, 1H, ArH), 8.05-8.07 (m, 2H, ArH), 8.71 (bs, 1H, NHCO); MS (ESI, m/z): $724.3[\mathrm{M}]^{+}$; anal. calcd. (\%) for $\mathrm{C}_{34} \mathrm{H}_{36} \mathrm{O}_{12} \mathrm{~N}_{4} \mathrm{~S}$ : C 56.35, H 5.01, N 7.73; found (\%): C 56.38, H 5.05, N 7.76. 9e: yield $68.0 \%$, IR $\left(\mathrm{KBr}, v_{\max }, \mathrm{cm}^{-1}\right): 3436,2919,1739,1613$, 1513, 1440, 1407, 1175; ${ }^{1} \mathrm{H}$ NMR $\left(\mathrm{CDCl}_{3}, 300 \mathrm{MHz}, \delta \mathrm{ppm}\right): 1.50-$ $3.38(\mathrm{~m}, 13 \mathrm{H}), 3.07$ (d, 3H, N-CH $), 3.67$ (s, 3H, MeO-1 ), 3.91 (s, 3H, MeO-2), 3.95 (s, 3H, MeO-3), 4.25-4.29 (t, $2 \mathrm{H}, J=6.0 \mathrm{~Hz}, \mathrm{OCH}_{2}$ ), 4.44-4.48 (t, $\left.2 \mathrm{H}, J=6.0 \mathrm{~Hz}, \mathrm{OCH}_{2}\right), 4.65-4.72(\mathrm{~m}, 1 \mathrm{H}, \mathrm{H}-7), 6.54$ (s, 1H, H-4), 7.27 (m, 2H, Ar-H), 7.48 (s, 1H), 7.61-7.66 (m, 2H, ArH), 7.74-7.79 (m,1H, ArH), 8.04-8.06 (m, 2H, ArH), 8.93 (bs, 1H, NHCO); MS (ESI, m/z): $752.4[\mathrm{M}]^{+}$; anal. calcd. (\%) for $\mathrm{C}_{36} \mathrm{H}_{40} \mathrm{O}_{12} \mathrm{~N}_{4} \mathrm{~S}: \mathrm{C} 57.44$, H 5.36, N 7.44; found (\%): C 57.55, H 5.40, N 7.49. 9f: yield $60 \%$, IR $\left(\mathrm{KBr}, \mathrm{v}_{\max }, \mathrm{cm}^{-1}\right): 3438,2929,1719,1623,1503,1445,1368,1145 ;{ }^{1} \mathrm{H}$ NMR $\left(\mathrm{CDCl}_{3}, 300 \mathrm{MHz}, \delta \mathrm{ppm}\right): 1.50-3.36(\mathrm{~m}, 9 \mathrm{H}), 3.07$ (d, $3 \mathrm{H}, \mathrm{N}-$ $\mathrm{CH}_{3}$ ), 3.66 (s, 3H, MeO-1), 3.90 (s, 3H, MeO-2), 3.96 (s, 3H, MeO-3), $4.35\left(\mathrm{~d}, 2 \mathrm{H}, J=5.4 \mathrm{~Hz}, \mathrm{OCH}_{2}\right), 5.15\left(\mathrm{~d}, 2 \mathrm{H}, J=5.7 \mathrm{~Hz}, \mathrm{OCH}_{2}\right), 4.65-$ 4.72 (m, 1H, H-7), 5.94-6.09 (m, $2 \mathrm{H},-\mathrm{CH}=\mathrm{CH}-), 6.55$ (s, 1H, H-4), 7.26 (m, 2H, Ar-H), 7.49 (s, 1H), 7.60-7.66 (m, 2H, ArH), 7.72-7.79 (m, 1H, ArH), 8.03-8.06 (m, 2H, ArH), 8.96 ( bs, 1H, NHCO); MS (ESI, m/z): $750.2[\mathrm{M}]^{+}$; anal. calcd. (\%) for $\mathrm{C}_{36} \mathrm{H}_{38} \mathrm{O}_{12} \mathrm{~N}_{4} \mathrm{~S}: \mathrm{C} 57.59, \mathrm{H}$ 5.10, N 7.46; found (\%): C 58.01, H 5.21, N 7.45. 9j: yield $65 \%$, IR $\left(\mathrm{KBr}, v_{\max }, \mathrm{cm}^{-1}\right): 3438,2932,1732,1633,1560,1454,1381,1169 ;{ }^{1} \mathrm{H}$ $\mathrm{NMR}\left(\mathrm{CDCl}_{3}, 300 \mathrm{MHz}, \delta \mathrm{ppm}\right): 1.50-3.37$ (m, 17H), 3.07 (d, 3H, N$\mathrm{CH}_{3}$ ), 3.65 (s, 3H, MeO-1), 3.90 (s, 3H, MeO-2), 3.94 (s, 3H, MeO-3), 4.24-4.28 (t, $\left.2 \mathrm{H}, J=6.0 \mathrm{~Hz}, \mathrm{OCH}_{2}\right), 4.44-4.48(\mathrm{t}, 2 \mathrm{H}, J=6.0 \mathrm{~Hz}$, $\left.\mathrm{OCH}_{2}\right), 4.64-4.72$ (m, 1H, H-7), 6.50 (s, 1H, H-4), 7.34 (m, 2H, Ar-H), 7.47 (s, 1H), 7.62-7.67 (m, 2H, ArH), 7.75-7.78 (m, 1H, ArH), 8.058.07 (m, 2H, ArH), 9.07 ( bs, 1H, NHCO); MS (ESI, m/z): $780.4\left[\mathrm{M}^{+}\right.$; anal. calcd. (\%) for $\mathrm{C}_{38} \mathrm{H}_{44} \mathrm{O}_{12} \mathrm{~N}_{4} \mathrm{~S}$ : C 58.45, H 5.68, N 7.18; found (\%): C 58.48, H 5.65, N 7.15.

16. J. Tatsuzaki, M. Taniguchi, K.F. Bastow, K. Nakagawa-Goto, S.L. MorrisNatschke, H. Itokawa, K. Baba and K.H. Lee, Bioorg. Med. Chem., 15, 6193 (2007). 